\title{
Electronic Energy Spectra and Wave Functions on the Square Fibonacci Tiling
}

\author{
SHAHAR EVEN-DAR MANDEL and RON LIFSHITZ \\ School of Physics and Astronomy, Raymond and Beverly Sackler Faculty \\ of Exact Sciences, Tel Aviv University, Tel Aviv 69978, Israel
}

May 15, 2005

\begin{abstract}
We study the electronic energy spectra and wave functions on the square Fibonacci tiling, using an off-diagonal tight-binding model, in order to determine the exact nature of the transitions between different spectral behaviors, as well as the scaling of the total bandwidth as it becomes finite. The macroscopic degeneracy of certain energy values in the spectrum is invoked as a possible mechanism for the emergence of extended electronic Bloch wave functions as the dimension changes from one to two.
\end{abstract}

\section{Background and Motivation}

We continue our initial studies [1] of the off-diagonal tight-binding hamiltonian on the square Fibonacci tiling [2], in order to gain a better quantitative understanding of the nature of the transitions between different spectral behaviors in this 2-dimensional $(2 d)$ quasicrystal. We also consider more carefully the transition of the spectrum from singular-continuous to absolutely continuous, in going from one to two dimensions, and the implications of this transition on the possible emergence of extended Bloch wave functions.

The square Fibonacci tiling is constructed by taking two identical grids - each consisting 
of an infinite set of lines whose inter-line spacings follow the well-known Fibonacci sequence of short $(S)$ and long $(L)$ distances - and superimposing them at a $90^{\circ}$ angle. This construction can be generalized, of course, to any quasiperiodic sequence as well as to higher dimensions. On this tiling we define an off-diagonal tight-binding hamiltonian with zero on-site energy, and hopping amplitudes: $t$ for vertices connected by short $(S)$ edges; 1 for vertices connected by long $(L)$ edges; and zero for vertices that are not connected by edges.

The 2-dimensional Schrödinger equation for this model is given by

$$
\begin{aligned}
& t_{n+1} \Psi(n+1, m)+t_{n} \Psi(n-1, m) \\
& \quad+t_{m+1} \Psi(n, m+1)+t_{m} \Psi(n, m-1)=E \Psi(n, m),
\end{aligned}
$$

where $\Psi(n, m)$ is the value of a $2 d$ eigenfunction on a vertex labeled by the two integers $n$ and $m$, and $E$ is the corresponding eigenvalue. The hopping amplitudes $t_{j}$ are equal to 1 or $t$ according to the Fibonacci sequence as described above, and we do not lose much by limiting ourselves to the case $t>1$, as the important features of the model depend on the ratio of the two hopping amplitudes.

With no additional assumptions other than the absence of diagonal hopping this 2-dimensional eigenvalue problem, as well as its higher dimensional versions, are all separable. This allows us to use the known solutions for the $1 d$ problem $[3,4]$ in order to construct the solutions in two and higher dimensions (as was done for similar models in the past $[5,6]$ ). Two-dimensional eigenfunctions can therefore be expressed as products

$$
\Psi_{i j}(n, m)=\psi_{i}(n) \psi_{j}(m)
$$

with eigenvalues

$$
E_{i j}=E_{i}+E_{j}
$$

where $\psi_{i}(n)$ and $\psi_{j}(n)$ are two of the eigenfunctions of the corresponding $1 d$ eigenvalue equation on the Fibonacci chain, with eigenvalues $E_{i}$ and $E_{j}$. Consequently, the $2 d$ density of states (DOS) is given by a convolution of two $1 d$ densities of states. 


\section{$2 \quad$ Study of the $2 d$ spectrum}

Although the spectrum of the $1 d$ model is always a Cantor-like set with zero bandwidth and an infinite number of bands, the $2 d$ spectrum exhibits different behavior for different ranges of the parameter $t$. For $t$ close to 1 the gaps in the $1 d$ spectrum are small and disappear as a result of the addition of spectra (3), producing an absolutely continuous $2 d$ spectrum. For $t \gg 1$ the $2 d$ spectrum retains the Cantor-like structure of the $1 d$ spectrum. For intermediate values of $t$ the number of bands tends to infinity but the total bandwidth remains finite and it is unclear whether the spectrum has an absolutely continuous part.

In order to study the exact structure of the $2 d$ spectrum - the total bandwidth and number of bands as a function of $t$ - one has to perform a convolution of two $1 d$ spectra. The numerical study of the $1 d$ spectrum is difficult for low values of $t(t \approx 1)$ for which the gaps are very small, and for $t \gg 1$ for which the bands are very small. Further numerical difficulties are encountered in the convolution process itself. The desired $2 d$ spectrum, consisting of the set of points for which the $2 d$ DOS is not zero, is given by

$$
\bigcup_{i, j=1}^{F_{N}}\left[a_{i}^{(N)}+a_{j}^{(N)}, b_{i}^{(N)}+b_{j}^{(N)}\right],
$$

where $\left[a_{i}^{(N)}, b_{i}^{(N)}\right]$ is the $i^{\text {th }}$ interval in the relevant $1 d$ spectrum of the $N^{t h}$ approximant, and $F_{N}$ is the $N^{t h}$ Fibonacci number. Because $F_{N} \sim \tau^{N}$ the number of intervals in the union (4) behaves like $\tau^{2 N}$, and although many of the intervals may overlap, any attempt to directly study the band structure and the DOS requires a construction of the whole set. The amount of memory required to store such sets makes exact calculation implausible for high values of $N$ regardless of the value of $t$. Luckily, the total bandwidth of the $2 d$ spectrum can be calculated without full knowledge of the DOS. This is done by examination of a set of uniformly distributed energies in the relevant range and testing for each energy whether it lies within one of the above intervals. For a sufficiently large set of energies this procedure yields the measure of the spectrum after proper normalization.

For given values of $N$ and $t$ the $1 d$ spectrum is a symmetric set of $F_{N}$ intervals within 


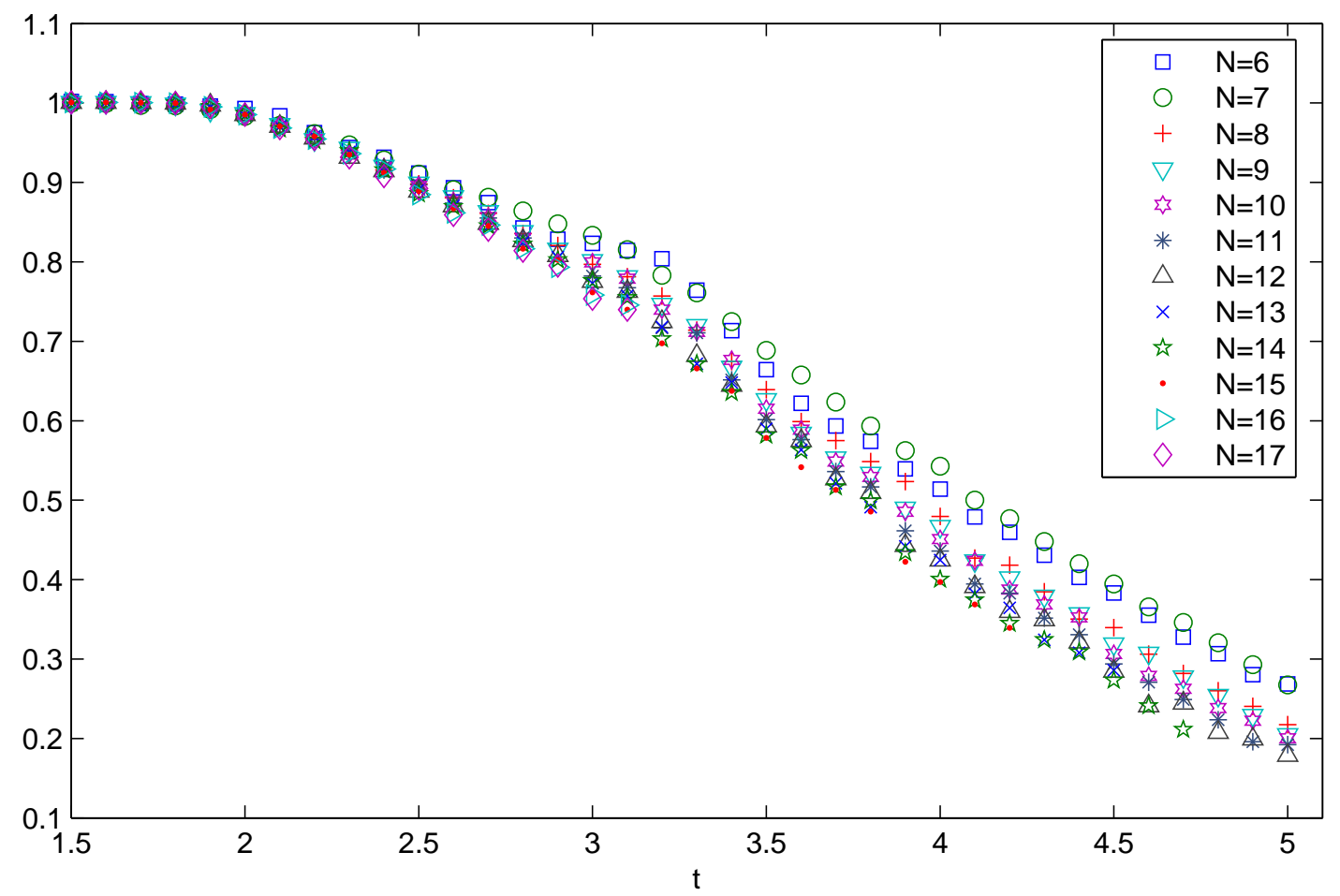

Figure 1: Total bandwidth of the $2 d$ spectrum divided by $4 r_{N}(t)$. For values of $t$ up to $\sim 2$ the spectrum consists of a single band regardless of $N$. At higher values the bandwidth clearly depends on $N$.

$\left[-r_{N}(t), r_{N}(t)\right]$, where $r_{N}(t)$ is the maximal eigenenergy of the $N^{t h} 1 d$ approximant. The $2 d$ spectrum is therefore a subset of the interval $\left[-2 r_{N}(t), 2 r_{N}(t)\right]$, which gives an upper bound of $4 r_{N}(t)$ on its total bandwidth. Fig. 1 shows the total bandwidth divided by $4 r_{N}(t)$ as a function of $t$ for a sequence of approximants $N=6 \ldots 17$. For values of $t$ up to $\sim 2$ the spectrum consists of a single band. For t's above 2 the normalized bandwidth becomes dependent on $N$. Using finite-size scaling (as suggested by Ashraff et al. [6]) we can obtain an estimate for the critical value $t_{c}$ at the transition between the intermediate and large $t$ regimes, where the total bandwidth vanishes. Fig. 2 shows the total bandwidth as a function of $1 / N$ for different values of $t>2$. Though some oscillatory behavior is observed, the behavior seems quite linear 


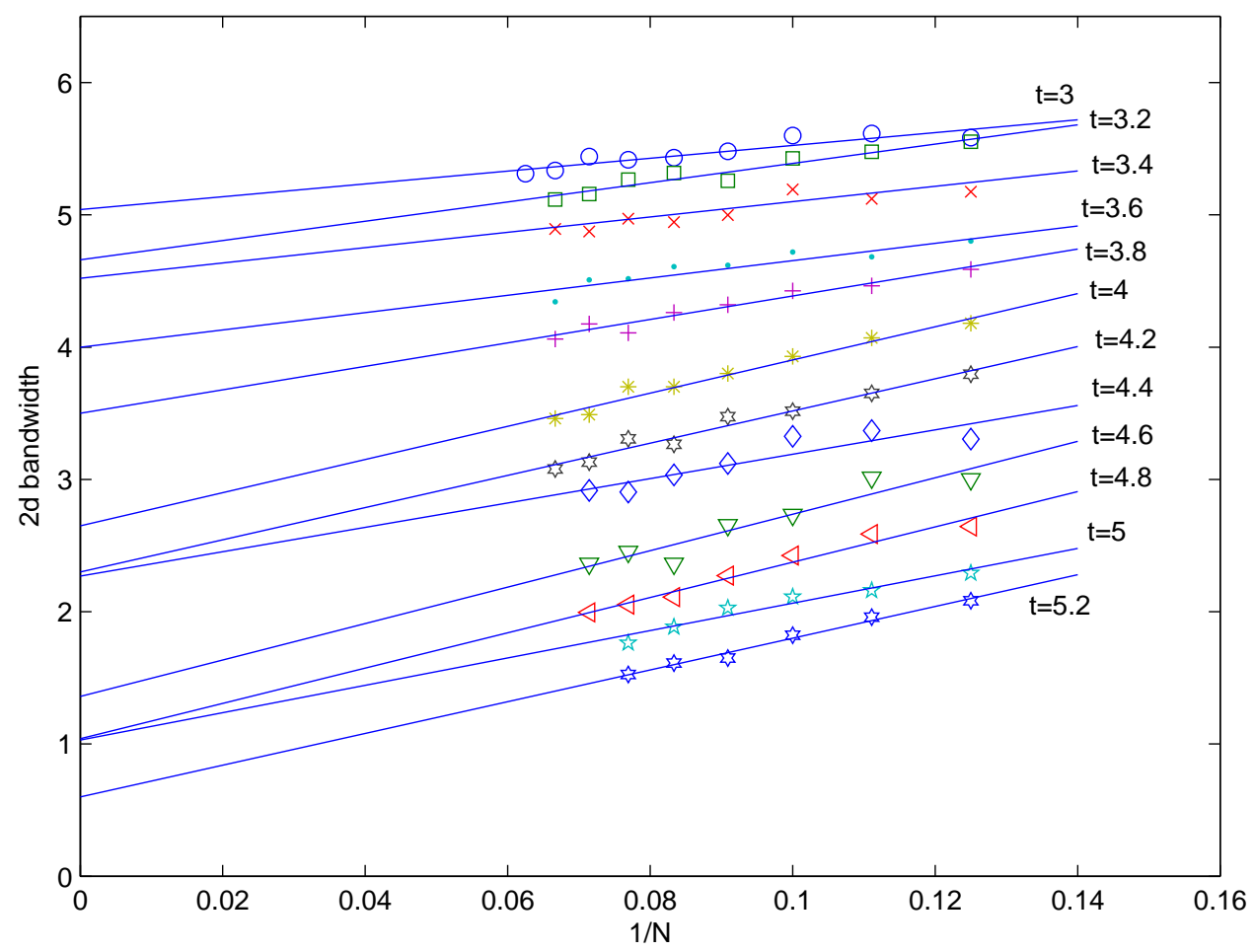

Figure 2: Total bandwidth as function of $1 / N$ for different values of $t$. Linear extrapolation to $1 / N \rightarrow 0$ gives a prediction for the total bandwidth at the infinite $N$ limit.

and extrapolation to $1 / N \rightarrow 0$ yields a prediction for the total bandwidth in the infinite $N$ limit. By plotting these estimates as a function of $t$ we obtain Fig. 3 which again exhibits an overall linear behavior. Extrapolation yields an estimate for a critical value of $t_{c} \simeq 5.375$ for the transition between the intermediate and large $t$ regimes.

It is worth noting that as $t$ is increased and the gaps grow, the degeneracy of energy levels decreases. At the asymptotic limit each interval in the $2 d$ spectrum should be exactly doubly degenerate (neglecting a single interval around $E=0$ ). When this limit is obtained the $2 d$ bandwidth should be approximately equal to $F_{N} B_{1 d}(t, N)$, where $B_{1 d}(t, N)$ is the bandwidth of the corresponding $1 d$ spectrum. The numerical data shows that this limit is to be obtained well beyond $t_{c}$. It is hence evident that the large $t$ regime of the $2 d$ spectrum is further divided 


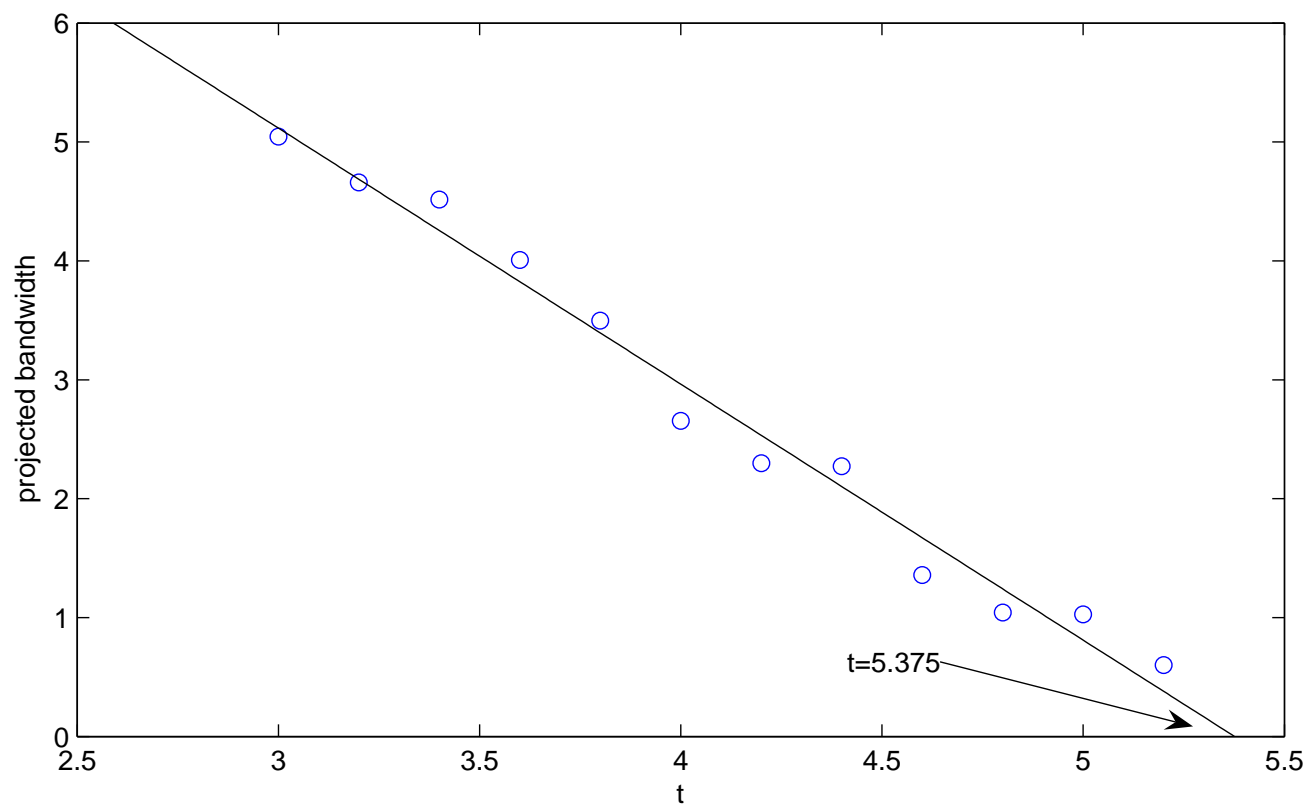

Figure 3: Extrapolation of the predicted infinite limit bandwidth to find the critical value $t_{c}$, above which the total bandwidth tends to zero.

into two regimes, one (at extremely large $t$ ) in which the energy levels are no-more than doubly degenerate, and another (at moderately large $t$ ) in which energy levels may have a higher degeneracy.

\section{$3 \quad$ Emergence of extended wave functions}

Since the eigenfunctions of the $1 d$ hamiltonian are critically localized it is clear that the $2 d$ eigenfunctions (2) should also be critically localized (Fig. 4(a-c)). However, the existence of a regime for $t$ close to 1 in which the spectrum, or at least a part of it, is absolutely continuous hints at the existence of extended wave functions. We proposed in the past [1] that the degeneracy of the $2 d$ spectrum, at values of $t$ close to 1 , may provide an explanation for the emergence of extended wave functions as the dimensionality increases.

To illustrate the possible role of degeneracy in the emergence of extended wave functions 

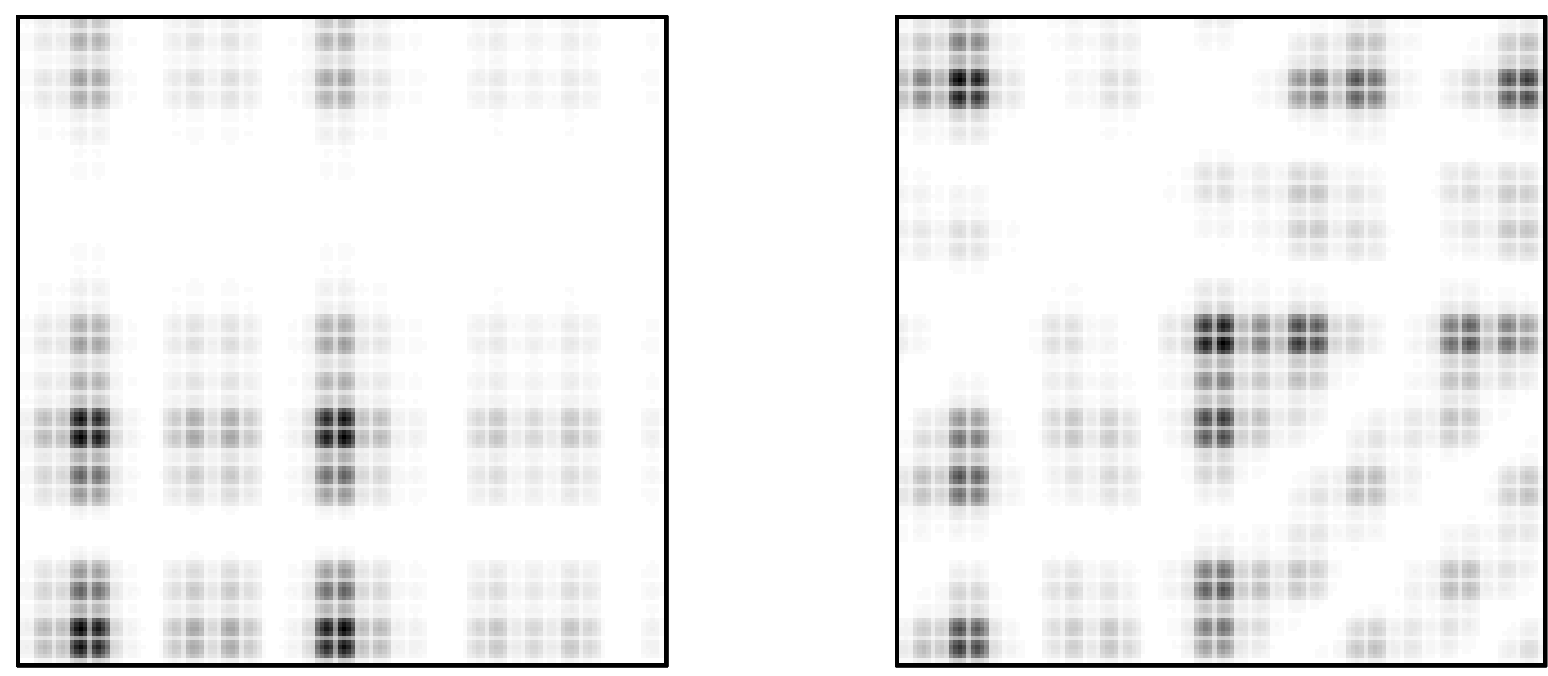

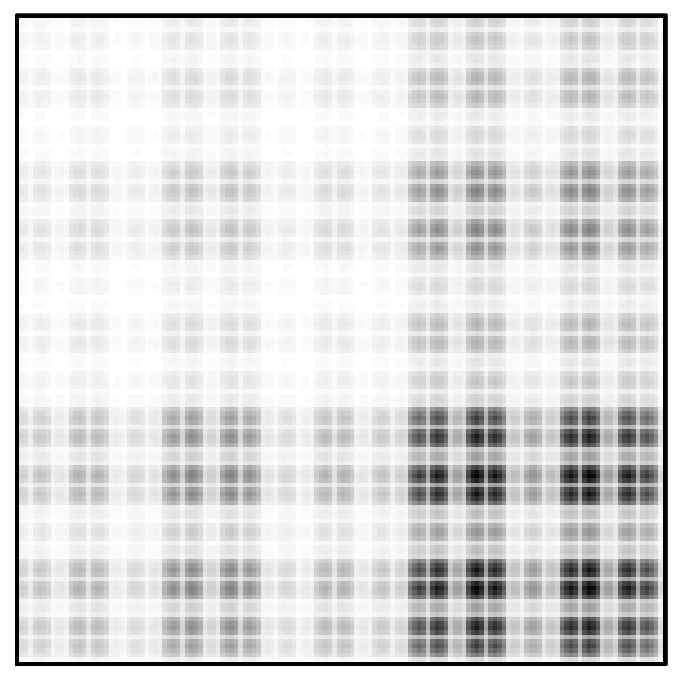

(c)

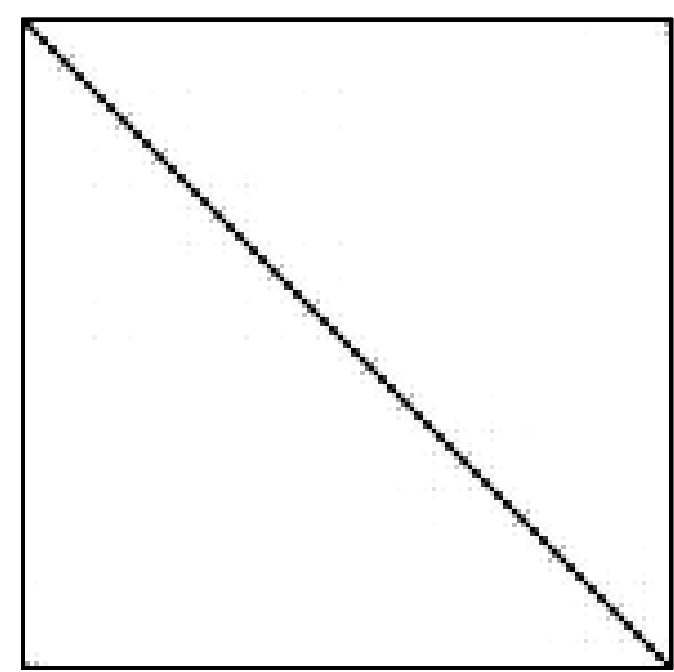

(d)

Figure 4: Wave functions on a single $144 \times 1442 d$ approximant. (a) A critical wave function $\Psi_{n, m}$; (b) A symmetrized critical wave function $\frac{1}{\sqrt{2}}\left(\Psi_{n, m}+\Psi_{m, n}\right)$; (c) A zero energy wave function $\Psi_{-n, n}$; and (d) A linear combination of zero energy wave functions producing an extended Bloch wave function along the diagonal, clearly showing the underlying Fibonacci modulation. 
we analyze the $E=0$ eigenfunction. These functions are macroscopically-degenerate for any value of $t$. A set of $1 d$ eigenfunctions can be found by directly diagonalizing the $1 d$ hamiltonian for a single approximant with periodic boundary conditions. The $F_{N}$ eigenfunctions obtained in this way generate $F_{N}^{2}$ eigenfunctions for the $2 d$ model, $F_{N}$ of which have $E=0$. These eigenfunctions, given by $\Psi_{i}(n, m)=(-1)^{m} \psi_{i}(n) \psi_{i}(m)$, are each critically localized, but we can demand that a linear combination $\sum_{i=1}^{N} a_{i} \Psi_{i}(n, m)$ of them have certain values on a given subset of sites by solving the equations for the coefficients $a_{i}$. Setting the values of a linear combination to be identical on half the points along the diagonal results in eigenfunctions of the form shown in Fig. 4(d). This wave-function is an extended Bloch function along the diagonal — clearly showing the underlying Fibonacci modulation-but is strongly localized in the perpendicular direction.

Whether the degeneracy of other energy values is sufficiently high to allow for a similar process to be carried out is not clear, but the chances for success should strongly improve as the tunnelling amplitude $t$ approaches 1 . This also corresponds to the regime in which the spectrum is (at least partly) absolutely continuous. For large $t$, where the spectrum is singular continuous, the degeneracy of levels other than $E=0$ is very small and no extended wave functions are expected to be found. Note that in 3 dimensions all the energies of the original $1 d$ spectrum are macroscopically degenerate. This means that extended wave-functions, similar to that of Fig. 4(d), can be constructed for a wider range of energies. Thus, as the dimensionality further increases so does the degeneracy of energy levels, and with it the ability to construct Bloch wave functions.

\section{Acknowledgments}

This research is supported by the Israel Science Foundation through Grant No. 278/00.

\section{References}

[1] R. Ilan, E. Liberty, S. Even-Dar Mandel, and R. Lifshitz, Ferroelectrics 305, 15 (2004).

[2] R. Lifshitz. J. of Alloys and Compounds 342, 186 (2002). 
[3] M. Kohmoto, L.P. Kadanoff, and C. Tang, Phys. Rev. Lett. 50, 1870 (1983); S. Ostlund, R. Pandit, D. Rand, H.S. Schellnhuber, and E.D. Siggia, Phys. Rev. Lett. 50, 1873 (1983); M. Kohmoto and J.R. Banavar, Phys. Rev. B 34, 563 (1986); and M. Kohmoto, B. Sutherland, and C. Tang, Phys. Rev. B 35, 1020 (1987).

[4] T. Janssen, in The Mathematics of Long-Range Aperiodic Order, ed. R.V. Moody, Kluwer, Dordrecht, 269 (1997); T. Fujiwara, in Physical Properties of Quasicrystals, ed. Z.M. Stadnik, Springer, Berlin, ch. 6 (1999); J. Hafner and M. Krajcí, ibid. ch. 7; and D. Damanik, in Directions in Mathematical Quasicrystals, ed. M. Baake and R.V. Moody, AMS, Providence, 277 (2000).

[5] K. Ueda and H. Tsunetsugu, Phys. Rev. Lett. 58, 1272 (1987); W.A. Schwalm and M.K. Schwalm, Phys. Rev. B 37, 9524 (1988); J.X. Zhong and R. Mosseri, J. Phys: Condens. Matter 7, 8383 (1995); and S. Roche and D. Mayou, Phys. Rev. Lett. 79, 2518 (1997); Yu.Kh. Vekilov, I.A. Gordeev, and E.I. Isaev, JETP 89, 995 (1999); Yu.Kh. Vekilov, E.I. Isaev, I.A. Gordeev, Mat. Sci. and Eng. 294-296, 553-555 (2000).

[6] J.A. Ashraff, J.-M. Luck, and R.B. Stinchcombe, Phys. Rev. B 41, 4314 (1990). 